Results Study is ongoing with 53/70 studies assessed. Early results suggest bicycle helmets were associated with reduced odds of head and facial injuries, with the strength of association greater for more severe head injuries. Stratification by injury type and severity reduced heterogeneity. Early analyses do not suggest publication bias and no time effects were found from 1998 onwards. Conclusions A systematic search of the literature is essential for meta-analysis, especially when assessing publication bias. Inadequate assessment of heterogeneity among included studies partly accounts for discrepancies in previously reported results. We found helmets were associated with significant reductions in head injury for cyclists injured in a crash.

\section{ALASKA YOUNG DRIVER SAFETY: DISTRACTED DRIVING, SEAT BELT USE AND DRINKING AND DRIVING INTERVENTIONS}

${ }^{1}$ Shannon Savage, ${ }^{2}$ Marcia Howell, ${ }^{2}$ Brian Saylor, ${ }^{1}$ Rhonda Johnson, ${ }^{1}$ Elizabeth Hodges Snyder. 'University of Alaska, Anchorage, USA; ${ }^{2}$ Alaska Injury Prevention Centre, USA

\subsection{6/injuryprev-2016-042156.249}

Background United States teenagers have the highest crash rate of any group in the nation. Alaska data tell a similar story. Leading causes of crashes for Alaska teen drivers are: driver inattention, unsafe speed, failure to yield and driver inexperience (Alaska Injury Prevention Centre, 2012). In partnership with the Alaska Injury Prevention Centre, a resource guide was created, listing best practices in Alaska teen driving interventions connected to three areas: distracted driving, seat belt use and drinking and driving.

Methods Guide content was evaluated for alignment with best practice through a multi-step filtering process. Available literature was distilled down to a final collection of safe teen driving intervention strategies based on best-available evidence. Results were categorised into a taxonomy of approaches, and were classified into levels of promise associated with certainty of effectiveness and potential population impact.

Results Strategies found to be most promising included public policy efforts surrounding graduated drivers' licensing programs, a minimum legal drinking age of 21 , cell phone restrictions while driving and seat belt requirements. In addition, community and parental roles of partnerships, boundary setting and monitoring teens' driving behaviours, were found to have equal levels of promise. Of significance was the importance of intervention strategies with diverse influences, including all levels of the Social Ecological Model.

Conclusions The developed process can be used as an effective model when synthesising large amounts of data, and can work in a variety of study areas to help practitioners understand complex research and guide them in their intervention choices. Resulting recommendations included multiple public policy enhancements in the state of Alaska, including graduated driver's license program modifications, enhancement of the state's zero-tolerance policy and broad scale restrictions of driver cell-phone use.

\section{PEDESTRIAN-VEHICLE INTERACTIONS: EARLY RESULTS FROM THE AUSTRALIAN NATURALISTIC DRIVING STUDY (ANDS)}

${ }^{1}$ Garrett Mattos, ${ }^{1}$ Raphael Grzebieta, ${ }^{1}$ Ann Williamson, ${ }^{2}$ Jake Olivier, ${ }^{1} J a n$ Eusebio, ${ }^{1}$ Wu Yi Zheng, ${ }^{3}$ John Wall, ${ }^{4}$ Jude Charlton, ${ }^{4,5}$ Mike Lenné, ${ }^{6}$ Jack Haley, ${ }^{7}$ Ben Barnes, ${ }^{8}$ Andry Rakotonirainy, ${ }^{9}$ Jeremy Woolley, ${ }^{1}$ Teresa Senserrick, ${ }^{4}$ Kristie Young, ${ }^{7}$ Narelle Haworth, ${ }^{1}$ Mike Regan, ${ }^{10}$ Samanatha Cockfield, ${ }^{11}$ David Healy, ${ }^{11}$ Antonietta Cavallo, ${ }^{11}$ Marilyn Di Stefano, ${ }^{12}$ Hee Loong Wong, ${ }^{13}$ lain Cameron, ${ }^{14}$ Michael Cornish, ${ }^{15}$ Christine Baird. ${ }^{1}$ Transport and Road Safety (TARS) Research, University of New South Wales, Australia; ${ }^{2}$ School of Mathematics and Statistics, University of New South Wales, Australia; ${ }^{3}$ Centre for Road Safety, Transport for NSW,Australia; ${ }^{4}$ Monash University Accident Research Centre (MUARC), Australia; ${ }^{5}$ Seeing Machines, Braddon, ACT,Australia; ${ }^{6}$ National Roads and Motorist Association (NRMA), Australia; ${ }^{7}$ Centre for Road Safety, Transport for NSW, Australia; ${ }^{8}$ Centre for Accident Research and Road Safety - Queensland (CARRSQ), Australia; ${ }^{9}$ Centre for Automotive Safety Research, University of Adelaide, Australia; ${ }^{10}$ Transport Accident Commission, Victoria, Australia; ${ }^{11}$ Vicroads, Australia; ${ }^{12}$ Hyundai Australia; ${ }^{13}$ Office of Road Safety, Main Roads Western Australia; ${ }^{14}$ Motor Accidents Commission, Adelaide; ${ }^{15}$ Motor Accidents Authority, Australia

\subsection{6/injuryprev-2016-042156.250}

Background Typologies have been defined previously for pedestrian-vehicle interactions and are primarily based on retrospective analysis of crash data. The naturalistic driving study currently underway in Australia makes it possible to study pedestrianvehicle interaction events that would not otherwise be identified in the crash data. This work evaluates the feasibility of using automated, manual, and semi-automated methods to identify pedestrian-vehicle interaction events.

Methods Sensors and cameras were installed on the vehicles of volunteers in and around two major Australian cities which recorded their natural driving behaviour for 4 months. Forward video from select vehicles was reviewed independently by two reviewers to identify potential pedestrian-vehicle interaction events from which a typology of behaviours was formulated. These events served as the gold standard against which select automated and semi-automated methods of identification were assessed.

Results A prototype typology of pedestrian-vehicle interaction events was formulated using naturalistic driving data and categorised in terms of risk of being struck. Some case scenarios will be discussed. The feasibility of using select automated, semi-automated, and manual methods to identify these events was also evaluated.

Conclusions This work provides a first look at using Australian naturalistic driving data to study the interactions between vehicles and pedestrians. These findings will assist in the development of 